\title{
CURVES OF GENUS $g$ WHOSE CANONICAL MODEL LIES ON A SURFACE OF DEGREE $g+1$
}

\author{
GIANFRANCO CASNATI
}

(Communicated by Lev Borisov)

\begin{abstract}
Let $C$ be a non-hyperelliptic curve of genus $g$. We prove that if the minimal degree of a surface containing the canonical model of $C$ in $\check{\mathbb{P}}_{k}^{g-1}$ is $g+1$, then either $g \geq 9$ and $C$ carries exactly one $g_{4}^{1}$ or $7 \leq g \leq 15$ and $C$ is birationally isomorphic to a plane septic curve with at most double points as singularities.
\end{abstract}

\section{INTRODUCTION AND NOTATION}

Let $k$ be an algebraically closed field. Let $C$ be a curve (i.e. a smooth, connected, projective scheme of dimension 1) and denote by $g \geq 2$ its genus. The canonical system on $C$ gives rise to a natural morphism $\phi: C \rightarrow \check{\mathbb{P}}_{k}^{g-1}$. The morphism $\phi$ is either the double cover of a rational normal curve or it is an isomorphism. In the former case $C$ is said to be hyperelliptic; in the latter case $C_{c a n}:=\phi(C)$ is called the canonical model of $C$.

The study of the intrinsic properties of a non-hyperelliptic curve $C$ is strictly related to the description of the embedding $C_{c a n} \subseteq \check{\mathbb{P}}_{k}^{g-1}$.

We are interested in characterising curves $C$ such that $C_{\text {can }}$ lies on a surface $S \subseteq \check{\mathbb{P}}_{k}^{g-1}$ (i.e. an integral, projective scheme of $\operatorname{dimension} 2$ ) for which $\operatorname{deg}(S)$ is fixed. Due to the geometric characterisation of the degree, we know that $\operatorname{deg}(S) \geq$ $g-2$. It is thus natural to ask first what happens for low values of $\operatorname{deg}(S)$, say $g-2, g-1, g$, and so on.

The first result along such lines is the following well-known Babbage-EnriquesNoether-Petri Theorem (see e.g. $\mathrm{A}-\mathrm{C}-\mathrm{G}-\mathrm{H}]$, p. 131, when the characteristic of $k$ is 0 and $[\mathrm{SD}$ otherwise).

Theorem A. Let $C$ be a non-hyperelliptic curve of genus $g \geq 3$. Then $C_{\text {can }} \subseteq \check{\mathbb{P}}_{k}^{g-1}$ is contained in an integral surface $S \subseteq \check{\mathbb{P}}_{k}^{g-1}$ of degree $g-2$ if and only if exactly one of the following two conditions holds.

i) $C$ is trigonal (i.e. there exists a map $C \rightarrow \mathbb{P}_{k}^{1}$ which is finite of degree 3 ): in this case $S$ is a rational normal scroll.

ii) $g=5$ and $C$ is isomorphic to a plane quintic curve: in this case $S$ is a Veronese surface.

A second result is the following theorem (see $[\mathrm{B}-\mathrm{C}-\mathrm{N}]$ and the references therein).

Received by the editors March 21, 2011 and, in revised form, July 1, 2011.

2010 Mathematics Subject Classification. Primary 14N25; Secondary 14H51, 14H30, 14 N05.

Key words and phrases. Curve, canonical model, tetragonality.

This work was done in the framework of PRIN 'Geometria delle varietà algebriche e dei loro spazi di moduli', cofinanced by MIUR (COFIN 2008). 
Theorem B. Let $C$ be a non-hyperelliptic curve of genus $g \geq 5$. Then $C_{\text {can }} \subseteq \check{\mathbb{P}}_{k}^{g-1}$ is contained in an integral surface $S \subseteq \check{\mathbb{P}}_{k}^{g-1}$ of degree $g-1$ if and only if one of the three following conditions holds.

i) $C$ is bielliptic (i.e. there exists a double cover $C \rightarrow E$ onto an elliptic curve): in this case $S$ is a cone on an elliptic normal curve contained in a hyperplane of $\check{\mathbb{P}}_{k}^{g-1}$ with vertex not on $C_{\text {can }}$.

ii) $g \leq 10$ and $C$ is birationally isomorphic to a plane sextic carrying $10-g$, possibly infinitely near, double points as singularities: in this case $S$ is the anticanonical image of the blowup of $\mathbb{P}_{k}^{2}$ at such points.

iii) $g=9$ and $C$ is isomorphic to a smooth complete intersection inside $\mathbb{P}_{k}^{3}$ of an integral quadric $Q$ with an integral quartic surface: in this case $S$ is the anticanonical image of $Q$.

Both of the above results also rest on the classification of surfaces of degree $g-2$ and $g-1$ in $\mathbb{P}_{k}^{g-1}$. Such an approach is not optimal as $\operatorname{deg}(S)$ increases. In [CS] we succeed to prove Theorem $\mathrm{C}$ below, via an analysis of the linear system cut out on $S$ by the quadrics through $C_{c a n}$.

Theorem C. Let $C$ be a non-hyperelliptic curve of genus $g$. If the minimal degree of a surface $S \subseteq \check{\mathbb{P}}_{k}^{g-1}$ containing $C_{\text {can }}$ is $g$, then $7 \leq g \leq 12$ and $S$ is a conic bundle over $\mathbb{P}_{k}^{1}$ whose fibres cut out on $C$ a base-point-free $g_{4}^{1}$. The curve $C$ does not carry any other base-point-free $g_{4}^{1}$.

The aim of the present paper is to examine the next case, namely the case of curves $C$ such that $C_{\text {can }}$ lies on a surface $S \subseteq \check{\mathbb{P}}_{k}^{g-1}$ with $\operatorname{deg}(S)=g+1$. We restrict our study to the case of a field $k$ of characteristic 0 . A refinement of the techniques used for proving Theorem $\mathrm{C}$ above allow us to obtain the following.

Theorem D. Let $C$ be a non-hyperelliptic curve of genus $g$ over an algebraically closed field $k$ of characteristic 0 . If the minimal degree of a surface $S \subseteq \check{\mathbb{P}}_{k}^{g-1}$ containing the canonical model of $C$ is $g+1$, then exactly one of the two following conditions holds.

i) $7 \leq g \leq 15$ and $C$ is birationally isomorphic to a plane septic $D$ with exactly $15-g$ possibly infinitely near double points as singularities: in this case $C_{\text {can }}$ is contained in the surface $\widehat{S} \subseteq \check{\mathbb{P}}_{k}^{g-1}$ of degree $g+1$ obtained as the birational image of $\mathbb{P}_{k}^{2}$ via the map defined by the linear system of quartics through the double points of the plane model $D$ (such an $\widehat{S}$ will be called a weak Bordiga surface).

ii) $g \geq 9$ and $C$ is endowed with a unique $g_{4}^{1}$ : in this case $S$ is a conic bundle onto $\mathbb{P}_{k}^{1}$ whose fibres cut out on the canonical model of $C$ the aforementioned basepoint-free $g_{4}^{1}$. If $g \geq 16$ such a $g_{4}^{1}$ is composed with an involution on a curve of genus 2 .

Very recently the aforementioned Theorems B, C, D have been generalised in [C], where the author proves that if the canonical model of a curve $C$ of genus $g$ lies on a surface of degree $g+\kappa<2 g-3$ with $\kappa \geq-1$ and $(g, \kappa) \neq(10,-1)$, then either $g \leq 3 \kappa+12$ or $\kappa$ is odd and $C$ is the double cover of a curve of genus $(\kappa+3) / 2$. Moreover, also a careful characterisation of such curves is given therein, under the extra hypothesis that $S$ is smooth. 
The proof of Theorem $\mathrm{D}$ is quite long and has been divided into some steps from section 3 to section 6 . In section 2 we recall some facts regarding tetragonal curves which are used in the proof. At the end of section 6 we list some remarks and comments.

Notation. We work over an algebraically closed field $k$ of characteristic 0 .

If $V$ is a vector space, then we denote by $\mathbb{P}(V)$ the corresponding projective space. In particular, we set $\mathbb{P}_{k}^{n}:=\mathbb{P}\left(k^{n+1}\right)$.

Let $X \subseteq \mathbb{P}_{k}^{n}$ be a closed subscheme and let $\mathcal{L}$ be a sheaf on $X$. If $q \in H^{0}(X, \mathcal{L})$ we will denote by $D_{0}(q) \subseteq X$ its zero-scheme.

For other definitions, results and notation we always refer to $\mathrm{Ha}$.

\section{Covers of Degree 4}

We start this paper recalling some standard facts about covers of degree 4 . If $C$ is a curve, then a morphism $\varrho: C \rightarrow \mathbb{P}_{k}^{1}$ is a cover of degree 4 if it is finite, flat and $\operatorname{rk}\left(\varrho_{*} \mathcal{O}_{C}\right)=4$. There exists a natural exact sequence

$$
0 \longrightarrow \mathcal{O}_{\mathbb{P}_{k}^{1}} \stackrel{\varrho^{\#}}{\longrightarrow} \varrho_{*} \mathcal{O}_{C} \longrightarrow \check{\mathcal{E}} \longrightarrow 0,
$$

where $\check{\mathcal{E}}$ is a locally free $\mathcal{O}_{\mathbb{P}_{k}^{1}}$-sheaf of rank 3 called the Tschirnhausen module of $\varrho$.

Since every locally free $\mathcal{O}_{\mathbb{P}_{k}^{1}}$-sheaf splits, we can fix a decomposition $\mathcal{E} \cong$ $\bigoplus_{i=1}^{3} \mathcal{O}_{\mathbb{P}_{k}^{1}}\left(\alpha_{i}\right)$. The sequence above and Serre duality yields $g+3=\alpha_{1}+\alpha_{2}+\alpha_{3}$.

Theorem 2.1. Let $C$ be a curve of genus $g$ and $\varrho: C \rightarrow \mathbb{P}_{k}^{1}$ a cover of degree 4. There exists an embedding $i: C \hookrightarrow \mathbb{P}:=\mathbb{P}(\mathcal{E}) \stackrel{\pi}{\longrightarrow} \mathbb{P}_{k}^{1}$, a locally free $\mathcal{O}_{\mathbb{P}_{k}^{1}}$-sheaf $\mathcal{F}:=\bigoplus_{j=1}^{2} \mathcal{O}_{\mathbb{P}_{k}^{1}}\left(\beta_{j}\right)$ such that $\sum_{j=1}^{2} \beta_{j}=g+3$ and a morphism $\delta: \pi^{*} \mathcal{F} \stackrel{\delta^{k}}{\longrightarrow} \mathcal{O}_{\mathbb{P}}(2)$ such that $C=D_{0}(\delta)$ and $\varrho=\pi_{\mid D_{0}(\delta)}$. Moreover

$$
\omega_{C} \cong i^{*}\left(\mathcal{O}_{\mathbb{P}}(1) \otimes \pi^{*} \mathcal{O}_{\mathbb{P}_{k}^{1}}(-2)\right) .
$$

Conversely let $\mathcal{E} \cong \bigoplus_{i=1}^{3} \mathcal{O}_{\mathbb{P}_{k}^{1}}\left(\alpha_{i}\right)$ and $\mathcal{F} \cong \bigoplus_{j=1}^{2} \mathcal{O}_{\mathbb{P}_{k}^{1}}\left(\beta_{j}\right)$ be such that $\sum_{i=1}^{3} \alpha_{i}=$ $\sum_{j=1}^{2} \beta_{j}$, define $\mathbb{P}:=\mathbb{P}(\mathcal{E}) \stackrel{\pi}{\longrightarrow} \mathbb{P}_{k}^{1}$ and let $\delta: \pi^{*} \mathcal{F} \rightarrow \mathcal{O}_{\mathbb{P}}(2)$. If $\varrho=\pi_{\mid D_{0}(\delta)}$ is a cover, then it has degree 4 and the Tschirnhausen module of $\varrho$ is $\check{\mathcal{E}}$.

Proof. See [C-E], Theorem 2.1 and section 4.

The following Bertini-type theorem will be used.

Theorem 2.2. Let $\mathcal{E}:=\bigoplus_{i=1}^{3} \mathcal{O}_{\mathbb{P}_{k}^{1}}\left(\alpha_{i}\right)$ and $\mathcal{F}:=\bigoplus_{j=1}^{2} \mathcal{O}_{\mathbb{P}_{k}^{1}}\left(\beta_{j}\right)$ be such that $\sum_{i=1}^{3} \alpha_{i}=\sum_{j=1}^{2} \beta_{j}$. Define $\mathbb{P}:=\mathbb{P}(\mathcal{E}) \stackrel{\pi}{\longrightarrow} \mathbb{P}_{k}^{1}$ and

$\mathcal{U}:=\left\{\delta \in H^{0}\left(\mathbb{P}, \mathcal{O}_{\mathbb{P}}(2) \otimes \pi^{*} \breve{\mathcal{F}}\right) \mid D_{0}(\delta)\right.$ is smooth and $\pi_{\mid D_{0}(a, b)}$ is a cover of degree 4$\}$.

If $S^{2} \mathcal{E} \otimes \check{\mathcal{F}}$ is globally generated, then $\mathcal{U} \neq \emptyset$ and is open.

Proof. See [-E], Theorem 4.5.

Let $C$ be a curve, let $\varrho: C \rightarrow \mathbb{P}_{k}^{1}$ be a cover of degree 4 and fix decompositions $\mathcal{E} \cong \bigoplus_{i=1}^{3} \mathcal{O}_{\mathbb{P}_{k}^{1}}\left(\alpha_{i}\right), \alpha_{1} \leq \alpha_{2} \leq \alpha_{3}, \mathcal{F} \cong \bigoplus_{j=1}^{2} \mathcal{O}_{\mathbb{P}_{k}^{1}}\left(\beta_{j}\right), \beta_{1} \leq \beta_{2}$, of the associated sheaves.

The triple $\alpha:=\left(\alpha_{1}, \alpha_{2}, \alpha_{3}\right)$ is called the scrollar invariants of $\varrho$. The number $\tau:=\beta_{1}-4$ is called the tetragonality of $\varrho$. 
Remark 2.3. We list below some results about the tetragonality and the scrollar invariants of a cover that we will use in the next sections. We are assuming that $C$ is a curve of genus $g \geq 4$, endowed with a cover $\varrho: C \rightarrow \mathbb{P}_{k}^{1}$ of degree 4 with invariants $\alpha:=\left(\alpha_{1}, \alpha_{2}, \alpha_{3}\right)$ and $\tau$. Such invariants have already been studied in several places. Besides the aforementioned paper [C-E], we recall for the reader's benefit [Ch] and the two papers [Sch1, Sch2] and $\mathrm{Br}$, where $e_{1}=\alpha_{3}-2, e_{2}=\alpha_{2}-2, e_{3}=\alpha_{1}-2$, $b_{1}=g-5-\tau, b_{2}=\tau$.

The following assertions hold true.

(1) $1 \leq \alpha_{1} \leq g+3 / 3,-2 \leq \tau \leq \min \left\{(g-5) / 2,2\left(\alpha_{1}-2\right)\right\}$ (see [Cs], Proposition 2.5).

(2) If $C$ is non-hyperelliptic, then $\alpha_{1} \geq 2$ and $\tau \geq-1$ (see Section 6.3 of [Sch1]).

(3) If $C$ is non-hyperelliptic and $\tau=-1$, then either $C$ is trigonal or it is isomorphic to a smooth plane quintic. Moreover, $g \leq 6$ (see Section 6.3 of Sch1]).

(4) If $C$ is non-hyperelliptic and $\tau<(g-9) / 3$, then $\varrho$ is composed with an involution onto a curve of genus $\alpha_{1}-1$. Moreover, $\tau=2\left(\alpha_{1}-2\right)$ (see [Sch2], Corollary 4.2).

(5) If $C$ is neither hyperelliptic nor trigonal nor isomorphic to a smooth plane quintic and $\varrho^{\prime}: C \rightarrow \mathbb{P}_{k}^{1}$ is a second cover of degree 4 with tetragonality $\tau^{\prime}$, then $\tau=\tau^{\prime}=0$. If $C$ is not bielliptic, then $g \leq 9$ (Proposition 3.4 of [Cs] and the references therein).

The Picard group Pic $(\mathbb{P})$ of $\mathbb{P}$ is generated by the class $\xi$ of the tautological sheaf $\mathcal{O}_{\mathbb{P}}(1)$ and by the class $f$ of a fibre of $\pi$. Moreover, the Chern equation on $\mathbb{P}$ becomes

$$
\xi^{3}=(g+3) \xi^{2} f
$$

Since $\mathcal{F} \cong \mathcal{O}_{\mathbb{P}_{k}^{1}}(\tau+4) \oplus \mathcal{O}_{\mathbb{P}_{k}^{1}}(g-1-\tau)$, it follows that the section $\delta \in H^{0}\left(\mathbb{P}, \mathcal{O}_{\mathbb{P}}(2) \otimes\right.$ $\pi^{*} \check{\mathcal{F}}$ ) defining the cover $\varrho$ corresponds to a pair

$$
(a, b) \in H^{0}\left(\mathbb{P}, \mathcal{O}_{\mathbb{P}}(2) \otimes \pi^{*} \mathcal{O}_{\mathbb{P}_{k}^{1}}(-\tau-4)\right) \oplus H^{0}\left(\mathbb{P}, \mathcal{O}_{\mathbb{P}}(2) \otimes \pi^{*} \mathcal{O}_{\mathbb{P}_{k}^{1}}(\tau-g+1)\right) .
$$

In particular, $C \subseteq \mathbb{P}$ is the complete intersection of the two conic bundles $A:=$ $D_{0}(a) \in|2 \xi-(\tau+4) f|$ and $B:=D_{0}(b) \in|2 \xi-(g-1-\tau) f|$.

Due to Remark 2.3 (2) one deduces $\alpha_{1} \geq 2$; hence the linear system $|\xi-2 f|$ on $\mathbb{P}$ is globally generated and it defines a morphism $\phi: \mathbb{P} \rightarrow \Sigma:=\operatorname{im}(\phi) \subseteq \check{\mathbb{P}}_{k}^{g-1}$ onto a rational normal scroll. The restriction of $\phi$ to $C$ is exactly the canonical embedding (see Formula (2.1.1)). Moreover, $\phi$ maps the two conic bundles $A$ and $B$ onto two embedded surfaces $A_{c a n}$ and $B_{c a n}$ whose degrees are

$$
\begin{gathered}
\operatorname{deg}\left(A_{c a n}\right)=(2 \xi-(\tau+4) f)(\xi-2 f)^{2}=2 g-6-\tau, \\
\operatorname{deg}\left(B_{c a n}\right)=(2 \xi-(g-1-\tau) f)(\xi-2 f)^{2}=g-1+\tau .
\end{gathered}
$$

Since $\tau \leq(g-5) / 2$, it follows that $\operatorname{deg}\left(B_{\text {can }}\right) \leq \operatorname{deg}\left(A_{\text {can }}\right)$.

Notice that the planes in $\mathbb{P}$ cutting the $g_{4}^{1}$ on $C$ are mapped onto planes in $\Sigma$. In particular, the divisors of the $g_{4}^{1}$ are cut on $C_{c a n}$ by suitable planes. Each such a divisor is the base locus of a suitable pencil of conics in those planes, namely the pencil generated by the sections of $A_{\text {can }}$ and $B_{\text {can }}$.

The above discussion and Theorem 3.3 of [Cs] prove the following result.

Theorem 2.4. Let $C$ be a non-hyperelliptic curve of genus $g \geq 4, \varrho: C \rightarrow \mathbb{P}_{k}^{1}$ a cover of degree 4 with tetragonality $\tau$. 
Then the $g_{4}^{1}$ on $C$ corresponding to $\varrho$ is cut out on $C_{\text {can }}$ by a family of planes in $\mathbb{P}_{k}^{g-1}$ whose union $\Sigma$ is a scroll over $\mathbb{P}_{k}^{1}$. Moreover, $C_{\text {can }}$ is the complete intersection inside $\Sigma$ of two (in general non-uniquely determined) conic bundles $A_{\text {can }}$ and $B_{\text {can }}$ of respective degrees $2 g-6-\tau$ and $g-1+\tau$. If $\tau \geq 1$, then $\Sigma$ is smooth.

Finally the minimal degree of an integral surface $S \subseteq \Sigma$, different from $B_{\text {can }}$ and containing the canonical model $C_{\text {can }}$ of $C$ is $2 g-6-\tau$.

\section{A technical Lemma}

From now on, $C$ will denote a non-hyperelliptic curve of genus $g$. The canonical map $\phi: C \rightarrow \check{\mathbb{P}}_{k}^{g-1}$ is an embedding. We will assume that the minimal degree of a surface $S \subseteq \check{\mathbb{P}}_{k}^{g-1}$ containing $C_{c a n}$ is $g+h$.

Since $S$ is necessarily non-degenerate in $\check{\mathbb{P}}_{k}^{g-1}$, it follows that $h \geq-2$. As explained in the Introduction, the cases $-2 \leq h \leq 0$ have been examined in several papers.

Thus we will assume $h \geq 1$, from now on. In particular, $C$ is not trigonal (see Theorem A); thus $C_{c a n}$ is an ideal-theoretic intersection of quadrics. The cones projecting $C_{c a n}$ from one of its points have trivially degree $2 g-3$; thus we deduce that $h \leq g-3$. In $[\mathrm{C}-\mathrm{H}]$ it has been proved that equality actually occurs for $C$ general in its moduli space and $g \geq 23$.

Each quadric through $C_{c a n}$ cuts out on $S$, residually to $C_{c a n}$, a curve $\Gamma$ such that $\operatorname{deg}(\Gamma)=2 \operatorname{deg}(S)-\operatorname{deg}\left(C_{c a n}\right)=2 h+2$. If $C_{c a n} \subseteq \Gamma$ (e.g. if $\left.C_{c a n} \subseteq \operatorname{Sing}(S)\right)$, then $2 h+2=\operatorname{deg}(\Gamma) \geq \operatorname{deg}\left(C_{\text {can }}\right)=2 g-2$, a contradiction, since $h \leq g-3$. Thus $C_{\text {can }} \nsubseteq \Gamma$ (in particular, $C_{\text {can }} \nsubseteq \operatorname{Sing}(S)$ ).

Let us consider a minimal desingularisation $p: \widetilde{S} \rightarrow S$ (see [Za]). Let $\widetilde{C}$ be the strict transform of $C_{c a n}$ in $\widetilde{S}$ and denote by $H$ the total transform of a general hyperplane in $\check{\mathbb{P}}_{k}^{g-1}$. The linear system $|2 H-\widetilde{C}|$ on $\widetilde{S}$ has a fixed part $F$ and a movable part $|M|$. In particular, we have the linear equivalence $2 H \sim \widetilde{C}+M+F$. We have $H \cdot M=\operatorname{deg}(\Gamma)=2 h+2$, whence

$$
4 h+4=2 H \cdot M=\widetilde{C} \cdot M+M^{2}+F \cdot M,
$$

where $\widetilde{C} \cdot M, M^{2}, F \cdot M$ are all non-negative.

Lemma 3.2. With the notation above,

$$
h^{0}\left(\widetilde{C}, \mathcal{O}_{\widetilde{C}}(M)\right) \geq h^{0}\left(\widetilde{S}, \mathcal{O}_{\widetilde{S}}(M)\right) \geq h+2 .
$$

Proof. Since $2 g-2=\widetilde{C} \cdot H>M \cdot H=2 h+2$, it follows that $h^{0}\left(\widetilde{S}, \mathcal{O}_{\widetilde{S}}(M-\widetilde{C})\right)=0$. Thus the cohomology of the exact sequence

$$
0 \longrightarrow \mathcal{O}_{\widetilde{S}}(M-\widetilde{C}) \longrightarrow \mathcal{O}_{\widetilde{S}}(M) \longrightarrow \mathcal{O}_{\widetilde{C}}(M) \longrightarrow 0
$$

and $[\mathrm{C}-\mathrm{H}$, Lemma 2.5 (i)] yield inequalities (3.2.1).

The following result is now immediate.

Proposition 3.3. Let $C$ be a non-hyperelliptic curve of genus $g$ and let $g+h$ ( $h \geq 1$ ) be the minimal degree of a surface $S \subseteq \check{\mathbb{P}}_{k}^{g-1}$ containing $C_{\text {can }}$. Denote by $p: \widetilde{S} \rightarrow S$ a minimal resolution of singularities of $S, \widetilde{C} \subseteq \widetilde{S}$ the proper transform of $C_{c a n}$ and $|M|$ the movable part of $\left|p^{*} \mathcal{O}_{S}(2) \otimes \mathcal{O}_{\widetilde{S}}(-\widetilde{C})\right|$.

Then $\left|\mathcal{O}_{\widetilde{C}}(M)\right|$ contains a base-point-free $g_{d}^{h+1}$ with $2 h+3 \leq d \leq 4 h+4$. 
Proof. Due to the above lemma, the existence of a base-point-free $g_{d}^{h+1}$ contained in $\left|\mathcal{O}_{\widetilde{C}}(M)\right|$ with $d \leq 4 h+4$ is clear. If $d \leq 2 h+2$, then such a $g_{d}^{h+1}$ would be special, since $h \leq g-3$. This last fact and inequalities (3.2.1) contradict Clifford's Theorem, since $C$ is non-hyperelliptic and $2 h+2 \leq 2 g-4$. It follows that $d \geq 2 h+3$.

From now on, let $h=1$ : we are thus assuming that the minimal degree of a surface $S \subseteq \check{\mathbb{P}}_{k}^{g-1}$ containing $C_{c a n}$ is $g+1$. Thanks to Proposition 3.3, $C$ carries at least a possibly non-complete $g_{d}^{2}$ with $5 \leq d \leq \widetilde{C} \cdot M \leq 8$.

Lemma 3.4. Let $C$ be a non-hyperelliptic curve of genus $g$ and let $g+1$ be the minimal degree of a surface $S \subseteq \check{\mathbb{P}}_{k}^{g-1}$ containing $C_{c a n}$. Then $g \geq 7$ and $C$ does not carry any $g_{d}^{2}$ with $d \leq 6$.

Proof. If $g \leq 4$, then $C$ is trigonal; thus the minimal degree of a surface containing $C_{c a n}$ would be $g-1$, thanks to Theorem A. It follows that $g \geq 5$. If $g \leq 6$, then $C$ carries a $g_{4}^{1}$. Its tetragonality is at most $\tau=0$, thanks to Remark 2.3 (1). Theorem 2.4 yields the existence of a surface of degree at most $g-1$ containing $C_{c a n}$, contradicting the hypothesis of Theorem D. Thus, necessarily, $g \geq 7$.

Consider the morphism $f: C \rightarrow \mathbb{P}_{k}^{2}$ associated to the $g_{d}^{2}$. Its image $D \subseteq$ $\mathbb{P}_{k}^{2}$ is a non-degenerate integral curve and $d=\operatorname{deg}(f) \operatorname{deg}(D)$. If $d=5$, then $(\operatorname{deg}(f), \operatorname{deg}(D))=(1,5)$; i.e., $f$ is necessarily birational. Thus $g \leq p_{a}(D)=6$, a contradiction, since $C$ has genus $g \geq 7$. Thus, the case $d=5$ does not occur.

If $d=6$, we have three possible cases for the pair $(\operatorname{deg}(f), \operatorname{deg}(D))$, namely $(1,6)$, $(2,3),(3,2)$. In the last case $D$ is a conic; thus $C$ would be trigonal and hence $C_{c a n}$ would be contained on a surface of degree $g-2$ by Theorem A.

In the second case $D$ is a plane cubic; thus it is either rational or elliptic, whence $C$ turns out to be either hyperelliptic or bielliptic. The former case is a priori excluded and the latter is not possible since, otherwise, $C_{c a n}$ would be contained on a surface of degree $g-1$ by Theorem B.

Thus $f$ is birational and $D$ is a plane sextic. Again by Theorem B we deduce that also this case cannot occur. It follows that also the case $d=6$ does not occur.

Let $C$ be a non-hyperelliptic curve of genus $g$ and let $g+1$ be the minimal degree of a surface $S \subseteq \check{\mathbb{P}}_{k}^{g-1}$ containing $C_{c a n}$. Due to Proposition 3.3 and Lemma 3.4, we know that it carries at least a base-point-free $g_{d}^{2}$ with $7 \leq d \leq 8$. Such a $g_{d}^{2}$ is the movable part of $\left|p^{*} \mathcal{O}_{S}(2) \otimes \mathcal{O}_{\widetilde{S}}(-\widetilde{C})\right|$. It is trivial to check that each $g_{d}^{2}$ on $\mathrm{C}$ with $d \leq 8$ is special. Thus, Clifford's Theorem yields that such a $g_{d}^{2}$ can be contained in a $g_{d}^{3}$ at most.

\section{Curves with a Base-Point-Free $g_{7}^{2}$}

Let $C$ be endowed with a base-point-free $g_{7}^{2}$. We can assume that such a $g_{7}^{2}$ is actually complete. If not $C$ would be endowed with a $g_{7}^{3}$. Fixing a point $p_{0} \in C$ we thus would obtain, residually to $p_{0}$, a $g_{6}^{2}$ on $C$, which is not possible (see Lemma 3.4). Thus such a $g_{7}^{2}$ is actually complete and it defines a morphism $f: C \rightarrow \mathbb{P}_{k}^{2}$ which is birational onto its image $D \subseteq \mathbb{P}_{k}^{2}$, which is a non-degenerate integral curve of degree 7 .

Lemma 4.1. The curve $D$ carries at most a single triple point.

Proof. Since $C$ cannot be trigonal (due to the hypothesis and Theorem A), it follows that $D$ carries at most triple points. Let $p_{1}$ and $p_{2}$ be triple points on $D$. If $p_{2}$ is 
not infinitely near to $p_{1}$, then $C$ would be endowed with two distinct $g_{4}^{1}$. Due to Remark $2.3(5)$, each of them would have tetragonality $\tau=0$. Thus Theorem 2.4 would yield that $C_{c a n}$ would lie on a surface of degree $g-1$, again a contradiction.

It follows that $p_{2}$ is necessarily infinitely near to $p_{1}$; hence the curve $D$ has a single tangent at $p_{1}$. Let $(x, y)$ be affine coordinates such that $p_{1}=(0,0)$ and the tangent line at $p_{1}$ is $\{y=0\}$. Thus the equation of $D$ has necessarily the form

$$
f(x, y):=y^{3}+f_{4}(x, y)+f_{5}(x, y)+f_{6}(x, y)+f_{7}(x, y),
$$

where $f_{h}:=\sum_{i=0}^{h} a_{i}^{(h)} x^{h-i} y^{i} \in k[x, y]$ is a form of degree $h$. The strict transform of $D$ after the blowup $y=x u$ has equation

$$
u^{3}+x f_{4}(1, u)+x^{2} f_{5}(1, u)+x^{3} f_{6}(1, u)+x^{4} f_{7}(1, u),
$$

so that $p_{2}$ is the point $(0,0)$ in the $(x, u)$ coordinates. If such a point is triple, then $a_{0}^{(4)}=a_{1}^{(4)}=a_{0}^{(5)}=0$, whence we infer that

$$
\begin{gathered}
f_{4}(x, y)=a_{2}^{(4)} x^{2} y^{2}+a_{3}^{(4)} x y^{3}+a_{4}^{(4)} y^{4} \\
f_{5}(x, y)=a_{1}^{(5)} x^{4} y+a_{2}^{(5)} x^{3} y^{2}+a_{3}^{(5)} x^{2} y^{3}+a_{4}^{(5)} x y^{4}+a_{5}^{(5)} y^{5} .
\end{gathered}
$$

The lines through $p_{1}$ cut out a $g_{4}^{1}$ on $C$; thus they define a cover $\varrho: C \rightarrow \mathbb{P}_{k}^{1}$ with scrollar invariants $\left(\alpha_{1}, \alpha_{2}, \alpha_{3}\right)$ and tetragonality $\tau$. We have

$$
h^{0}\left(C, \varrho^{*} \mathcal{O}_{\mathbb{P}_{k}^{1}}(2)\right)=h^{0}\left(\mathbb{P}_{k}^{1}, \mathcal{O}_{\mathbb{P}_{k}^{1}}(2)\right)+\sum_{i=1}^{3} h^{0}\left(\mathbb{P}_{k}^{1}, \mathcal{O}_{\mathbb{P}_{k}^{1}}\left(2-\alpha_{i}\right)\right) .
$$

The integral conic $\widehat{D}$ with equation $y-x^{2}$ intersects $D$ at $p_{1}$ with multiplicity 6 ; hence it defines a divisor in $\left|\varrho^{*} \mathcal{O}_{\mathbb{P}_{k}^{1}}(2)\right|$ which cannot be cut out by the union of two lines through $p_{1}$. It follows that $h^{0}\left(C, \varrho^{*} \mathcal{O}_{\mathbb{P}_{k}^{1}}(2)\right) \geq 4$; hence $\alpha_{1} \leq 2$. We conclude that $\tau \leq 2\left(\alpha_{1}-2\right) \leq 0$. Arguing as in the previous case of two distinct triple points we have a contradiction.

Let $p_{1}, \ldots, p_{r}$ be the singular (possibly infinitely near) points of $D$. We assume that $p_{1} \in \mathbb{P}_{k}^{2}$, and we can assume that $p_{2}$ is in the blowup $\widetilde{S}_{1}$ of $\mathbb{P}_{k}^{2}$ at $p_{1}$, that $p_{3}$ is in the blowup $\widetilde{S}_{2}$ of $\widetilde{S}_{1}$ at $p_{2}$, and so on. We denote by $q: \widetilde{S}:=\widetilde{S}_{r} \rightarrow \mathbb{P}_{k}^{2}$ the composition of such a sequence of blowups. Let $e_{1}, \ldots, e_{r}$ be the total transforms in $\widetilde{S}$ of the exceptional divisors of $\widetilde{S}_{1}, \ldots, \widetilde{S}_{r}$; hence $e_{i}^{2}=-1$. Let $\ell$ be the pull-back of a general line of $\mathbb{P}_{k}^{2}$. Finally let $D_{0}:=D$ and $D_{i} \subseteq \widetilde{S}_{i}$ be the strict transform of $D_{i-1}$ for $i=1, \ldots, r$. We start by recalling the following definition (see [De, Séminaire III, Définition 1).

Definition 4.2. With the above notation, the points $p_{1}, \ldots, p_{r}$ are said to be in almost general position if the following conditions hold.

i) No four among them are on the same line.

ii) No seven among them are on the same conic.

iii) For each $j=1, \ldots, r-1$ the point $p_{j+1} \in \widetilde{S}_{j}$ does not lie on any proper transform $\widehat{e_{i}}$ of $e_{i}, i=1, \ldots, j$, such that ${\widehat{e_{i}}}^{2}=-2$.

Lemma 4.3. The curve $D$ carries at most double points as singularities.

Proof. Assume that $D$ carries a triple point $p_{1}$, the other singularities $p_{2}, \ldots, p_{r}$ being double points due to Lemma 4.1 above. Since $g \geq 7$, it follows that $r \leq 6$. We first show that the points $p_{1}, \ldots, p_{r}$ are in almost general position. 
The lines through $p_{1}$ induce a base-point-free $g_{4}^{1}$ on $C$. If $g \leq 8$, due to Remark 2.3 (1) the tetragonality $\tau$ of such a $g_{4}^{1}$ would be at most 1 , a contradiction thanks to Theorem 2.4; thus $g \geq 9$.

Since $g \geq 9$, we have $r \leq 6$. We claim that the points $p_{1}, \ldots, p_{r}$ are in almost general position. Indeed, in our setup, condition ii) is automatically satisfied. Since the points $p_{1}, \ldots, p_{r}$ are singular points of a plane septic, it follows that condition i) is trivially satisfied too. It remains to examine condition iii). Let $p_{i}$ be a double point and let $p_{i+1}$ be an infinitely near (necessarily double) point. We have $D_{i+1}=$ $q_{i}^{*} D_{i}-2 e$, where $q_{i}: \widetilde{S}_{i+1} \rightarrow \widetilde{S}_{i}$ is the blowup map at $p_{i}$ and $e$ its exceptional divisor. In particular, $e \cdot D_{i+1}=2$; thus the intersection of $D_{i+1}$ and $e$ at the double point $p_{i+1}$ is transversal. Blowing up again, the new double point infinitely near to $p_{i+1}$, if any, cannot lie on the strict transform of $e$. It follows that condition iii) is satisfied for the points $p_{i}$ that are not infinitely near to $p_{1}$.

Now we fix our attention on the point $p_{1}$. If the triple point $p_{1}$ has at least two tangent lines, then one can trivially repeat the above argument. The only non-trivial case that we have to examine is the case of a plane septic with a triple point with one tangent line. If this is the case, then the equation of $D$ is of the form (4.1.1), in a suitable affine neighbourhood of $p_{1}=(0,0)$. After the blowup $y=x u$, the strict transform of $D$ has an equation of the form (4.1.2). Again the point $p_{2}$ is $(0,0)$ in the coordinates $(x, u)$. Since it is assumed to be a double point, we have $a_{0}^{(4)}=0$. If $a_{1}^{(4)} \neq 0$, it follows that $p_{2}$ is a node; thus condition iii) above is trivially satisfied. If $a_{1}^{(4)}=0$, then necessarily $a_{0}^{(5)} \neq 0$, since $p_{2}$ is a double point. It follows that $p_{2}$ is an ordinary cusp, whence again condition iii) is satisfied.

The strict transform $\widetilde{D}$ of $D$ on $\widetilde{S}$ is in the linear system $\left|7 \ell-3 e_{1}-2 e_{2}-\cdots-2 e_{r}\right|$ and, by adjunction on $\widetilde{S}$, the canonical system on $\widetilde{D}$ is cut out by the linear system $\left|4 \ell-2 e_{1}-e_{2}-\cdots-e_{r}\right|$. Such a linear system contains the divisors splitting as the sum of a divisor in $\left|3 \ell-e_{1}-e_{2}-\cdots-e_{r}\right|$ plus a divisor in $\left|\ell-e_{1}\right|$. Both such linear systems are free from fixed components (for the first one, see [De], Séminaire III, Théorème 1); thus $\left|4 \ell-2 e_{1}-e_{2}-\cdots-e_{r}\right|$ is free from fixed components as well.

Let $A \in\left|4 \ell-2 e_{1}-e_{2}-\cdots-e_{r}\right|$. Since $\mathcal{O}_{\widetilde{S}}(A-\widetilde{D}) \cong \omega_{\widetilde{S}}$ and $h^{i}\left(\widetilde{S}, \omega_{\widetilde{S}}\right)=$ $h^{2-i}\left(\widetilde{S}, \mathcal{O}_{\widetilde{S}}\right)=h^{2-i}\left(\mathbb{P}_{k}^{2}, \mathcal{O}_{\mathbb{P}_{k}^{2}}\right)$ (see [Ha], Proposition V.3.4), it follows that the cohomology of

$$
0 \longrightarrow \mathcal{O}_{\widetilde{S}}(A-\widetilde{D}) \longrightarrow \mathcal{O}_{\widetilde{S}}(A) \longrightarrow \mathcal{O}_{\widetilde{D}}(A) \longrightarrow 0
$$

yields

$$
h^{0}\left(\widetilde{S}, \mathcal{O}_{\widetilde{S}}\left(4 \ell-2 e_{1}-e_{2}-\cdots-e_{r}\right)\right)=h^{0}\left(\widetilde{D}, \mathcal{O}_{\widetilde{D}}(A)\right)=h^{0}\left(C, \omega_{C}\right)=g ;
$$

thus $\left|4 \ell-2 e_{1}-e_{2}-\cdots-e_{r}\right|$ induces a rational map $s: \widetilde{S} \rightarrow \mathbb{P}_{k}^{g-1}$. Let $\widehat{S}$ be the closure of the image of $s$. Clearly $C_{\text {can }} \subseteq \widehat{S}$. Since the divisors in $\mid 4 \ell-2 e_{1}-e_{2}-$ $\cdots-e_{r} \mid$ cut out on $D$ the canonical divisor, it follows that such divisors are mapped on curves via $s$, which are necessarily different from $C_{c a n}$. We conclude that $\widehat{S}$ must be a surface of degree $\operatorname{deg}(\widehat{S}) \leq\left(4 \ell-2 e_{1}-e_{2}-\cdots-e_{r}\right)^{2}=g$, contradicting the hypothesis. It follows that $D$ cannot carry triple points.

Up to now we have proved that the plane model $D$ of $C$ is a septic curve endowed with $r:=15-g$ possibly infinitely near double points $p_{1}, \ldots, p_{r}$. As above the canonical system on $\widetilde{D}$ is cut out by the linear system $\left|4 \ell-e_{1}-\cdots-e_{r}\right|$ and again 
we have a natural rational map $s: \widetilde{S} \rightarrow \widehat{S} \subseteq \mathbb{P}_{k}^{g-1}$ whose image $\widehat{S}$ contains $C_{c a n}$. We claim that $s$ is birational.

To this purpose we examine the position of the points $p_{1}, \ldots, p_{r}$. Obviously condition i) of Definition 4.2 is satisfied since $\operatorname{deg}(D)=7$. Moreover, the same argument used before Lemma 4.3 shows that condition iii) holds as well. Assume that condition ii) is satisfied (which certainly occurs if $r \leq 6$ ). As in the proof of Lemma 4.3 we deduce that $\left|4 \ell-e_{1}-\cdots-e_{r}\right|$ is free from fixed components, that $\widehat{S}$ is a surface and that inequality

$$
\operatorname{deg}(s) \operatorname{deg}(\widehat{S}) \leq\left(4 \ell-e_{1}-\cdots-e_{r}\right)^{2}=g+1
$$

holds. Due to the hypothesis we deduce that $s$ is birational and that the above inequality is actually an equality; thus $\left|4 \ell-e_{1}-\cdots-e_{r}\right|$ is base-point-free.

Now assume that condition ii) does not hold (which implies $r=7,8$ ). Since $\operatorname{deg}(D)=7$, at most seven among the points $p_{1}, \ldots, p_{r}$ can actually lie on a conic. Assume that $\left|2 \ell-e_{1}-\cdots-e_{7}\right|$ contains an element $\Gamma$. The linear system $\mid 4 \ell-$ $e_{1}-\cdots-e_{r} \mid$ contains all the divisors splitting as the sum of $\Gamma$ plus a divisor in either $|2 \ell|$ (if $r=7$ ) or $\left|2 \ell-e_{8}\right|$ (if $r=8$ ). Such a second linear system has no fixed components; thus the only possible fixed component of $\left|4 \ell-e_{1}-\cdots-e_{r}\right|$ could be $\Gamma$. If this is the case the map $s$ coincides with the map induced by either $|2 \ell|$ (if $r=7$ ) or $\left|2 \ell-e_{8}\right|$ (if $r=8$ ), which is birational. It follows that $\widehat{S}$ is a surface and $\operatorname{deg}(\widehat{S}) \leq 4<g+1$, a contradiction, whence we deduce that $\left|4 \ell-e_{1}-\cdots-e_{r}\right|$ has no fixed components. Arguing as in the previous cases it turns out that $\widehat{S}$ is a surface and $\operatorname{deg}(\widehat{S})=g+1$. We can summarise the results of this section in the following.

Proposition 4.4. Let $C$ be a non-hyperelliptic curve of genus $g$ and let $g+1$ be the minimal degree of a surface $S \subseteq \widetilde{\mathbb{P}}_{k}^{g-1}$ containing $C_{\text {can }}$.

If $C$ carries a base-point-free $g_{7}^{2}$, then $7 \leq g \leq 15$; such a $g_{7}^{2}$ is complete and induces a birational morphism of $C$ onto a plane septic $D$ with exactly $15-g$ (possibly infinitely near) double points. The linear system of quartics through the double points of $D$ induces a birational map onto a surface $\widehat{S} \subseteq \mathbb{P}_{k}^{g-1}$ of degree $g+1$ and $C_{\text {can }} \subseteq \widehat{S}$.

The above discussion and the construction of the classical Bordiga surface of degree 6 in $\mathbb{P}_{k}^{4}$ yield naturally to calling such a surface $\widehat{S}$ a weak Bordiga surface (associated to $\left\{p_{1}, \ldots, p_{r}\right\}$ ).

\section{Curves With A BASE-Point-Free $g_{8}^{2}$}

With the notation of section 3 , let $|M|$ be the movable part of $\mid p^{*} \mathcal{O}_{S}(2) \otimes$ $\mathcal{O}_{\widetilde{S}}(-\widetilde{C}) \mid$. We know that $\left|\mathcal{O}_{\widetilde{C}}(M)\right|$ contains a base-point-free $g_{d}^{2}$ with $7 \leq d \leq 8$. The case when $C$ is endowed with a $g_{7}^{2}$ has been analysed in the previous section. Thus we will assume in this section that $d=8$.

We can assume that such a $g_{8}^{2}$ is actually complete since, otherwise, $C$ would be endowed with a $g_{8}^{3}$. Fixing a point $p_{0} \in C$ we thus would obtain, residually to $p_{0}$, a $g_{7}^{2}$ on $C$; hence we would be in the previous case $d=7$. Thus, from now on, we will assume that $C$ does not carry any $g_{7}^{2}$.

Recall that inequalities $(3.2 .1)$ give $h^{0}\left(\widetilde{S}, \mathcal{O}_{\widetilde{S}}(M)\right)=3$. Since $\widetilde{C} \cdot M=8$, it follows that $M^{2}=F \cdot M=0$. Thus $|M|$ is base-point-free; hence its general element $\bar{M}$ is 
smooth due to Bertini's Theorem. The cohomology of the exact sequence

$$
0 \longrightarrow \mathcal{O}_{\widetilde{S}} \longrightarrow \mathcal{O}_{\widetilde{S}}(M) \longrightarrow \mathcal{O}_{\bar{M}}(M) \longrightarrow 0
$$

gives $h^{0}\left(M, \mathcal{O}_{\bar{M}}(M)\right) \geq 2$. Since the restriction of $|M|$ to $\bar{M}$ is obviously effective and it has degree 0 , it follows that $\bar{M}$ has at least two connected components. Since $\bar{M} \cdot H=4$, it also follows that $\bar{M}$ has at most four connected components.

In what follows we will examine the possible configurations of the components of $\bar{M}$.

Lemma 5.1. With the above notation, let $M^{\prime}$ be a component of $\bar{M}$. Then $M^{\prime} \cdot \widetilde{C}=$ $2 M^{\prime} \cdot H$.

Proof. Due to the arbitrariness of the component $M^{\prime}$, it suffices to prove that it is not possible to have $M^{\prime} \cdot \widetilde{C}>2 M^{\prime} \cdot H$. Due to the hypothesis and Theorem A, we can exclude that the curve $C$ is either trigonal or isomorphic to a plane quintic; thus $C_{c a n}$ is an ideal-theoretic intersection of quadrics. Let $\Gamma^{\prime}:=p\left(M^{\prime}\right) \subseteq S$ and let $Q$ be any quadric containing $C_{c a n}$. Then $M^{\prime} \cdot H=\operatorname{deg}\left(\Gamma^{\prime}\right)$ and $\widetilde{C} \cdot M^{\prime}=\operatorname{deg}\left(C_{c a n} \cap \Gamma^{\prime}\right)$, whence

$$
2 M^{\prime} \cdot H=2 \operatorname{deg}\left(\Gamma^{\prime}\right)=\operatorname{deg}\left(Q \cap \Gamma^{\prime}\right) \geq \operatorname{deg}\left(C_{c a n} \cap \Gamma^{\prime}\right)=\widetilde{C} \cdot M^{\prime}>2 M^{\prime} \cdot H,
$$

a contradiction.

Lemma 5.2. Let $\bar{M}_{1}$ be an integral component of the aforementioned general divisor $\bar{M}$. Then $\bar{M}_{1} \cdot H \leq 2$.

Proof. Since $\bar{M}$ is not connected, it follows that $\bar{M}=\bar{M}_{1}+\bar{M}_{2}$, where $\bar{M}_{2} \neq 0$ is effective and $\bar{M}_{1} \cdot \bar{M}_{2}=0$. We have that $|M|$ has no fixed components; thus $\bar{M}_{i}^{2}=\bar{M} \cdot \bar{M}_{i} \geq 0$. Since $\bar{M}^{2}=\bar{M}_{1}^{2}+\bar{M}_{2}^{2}=0$, the vanishing $\bar{M}_{1}^{2}=\bar{M}_{2}^{2}=0$ follows.

Thus the subset of $|M|$ consisting of divisors containing $\bar{M}_{1}$ coincides with the sublinear system of $|M|$ of divisors through a fixed general point $P \in \bar{M}_{1}$. We conclude that

$$
h^{0}\left(\widetilde{S}, \mathcal{O}_{\widetilde{S}}\left(\bar{M}_{2}\right)\right)=h^{0}\left(\widetilde{S}, \mathcal{O}_{\widetilde{S}}(M)\right)-1 \geq 2 .
$$

Since $h^{0}\left(\widetilde{S}, \mathcal{O}_{\widetilde{S}}\left(\bar{M}_{2}-\widetilde{C}\right)\right)=0$, the exact sequence

$$
0 \longrightarrow \mathcal{O}_{\widetilde{S}}\left(\bar{M}_{2}-\widetilde{C}\right) \longrightarrow \mathcal{O}_{\widetilde{S}}\left(\bar{M}_{2}\right) \longrightarrow \mathcal{O}_{\widetilde{C}}\left(\bar{M}_{2}\right) \longrightarrow 0
$$

yields

$$
h^{0}\left(\widetilde{C}, \mathcal{O}_{\widetilde{C}}\left(\bar{M}_{2}\right)\right) \geq h^{0}\left(\widetilde{S}, \mathcal{O}_{\widetilde{S}}\left(\bar{M}_{2}\right)\right) \geq 2 .
$$

Due to Lemma 5.1 we have $\bar{M}_{2} \cdot \widetilde{C}=2$. Thus $C$ would be endowed with a pencil of divisor of degree 2 , a contradiction since $C$ is neither rational nor hyperelliptic. It follows that $\bar{M}_{1} \cdot H \leq 2$.

Lemma 5.3. With the above notation, $C$ carries a $g_{4}^{1}$ which is cut out on $C_{\text {can }}$ by a family of curves of degree 2 contained in $S$.

Proof. As above, we write $\bar{M}=\bar{M}_{1}+\bar{M}_{2}$, where $\bar{M}_{1}$ is integral and $\bar{M}_{1} \cdot \bar{M}_{2}=0$. We know from Lemma 5.2 that $\bar{M}_{1} \cdot H \leq 2$; i.e., the irreducible components of $\bar{\Gamma}:=p(\bar{M}) \subseteq S$ are either lines or integral conics.

Assume that $\bar{M}_{1}$ is mapped onto an integral conic. As in the proof of Lemma 5.2, $h^{0}\left(\widetilde{C}, \mathcal{O}_{\widetilde{C}}\left(\bar{M}_{2}\right)\right) \geq 2$ and $\bar{M}_{2} \cdot \widetilde{C}=4$; thus we obtain a $g_{4}^{1}$ which is cut out on $C_{\text {can }}$ by the images of the elements of $\left|\mathcal{O}_{\widetilde{C}}\left(\bar{M}_{2}\right)\right|$ which are curves on $S$ of degree 2 . The general curve of this linear system is either a conic or pair of skew lines. 
Now assume that all the components of $\bar{M}$ are mapped via $p$ on lines in $S$. In this case $\bar{M}$ has four components, say $\bar{M}_{i}, i=1, \ldots, 4$. Since $\bar{M}^{2}=0$ and $|M|$ has no fixed points or base components, again it follows that $\bar{M}_{i}^{2}=0, i=1, \ldots, 4$.

Let $p_{0}$ be a general point on $\widetilde{S}$. There are many divisors in $|M|$ containing $p_{0}$ but all such divisors must have a unique common component through $p_{0}$ whose image via $p$ is necessarily a line on $S$. Thus we can define naturally a rational map from $\widetilde{S}$, hence from the ruled surface $S$, onto the general hyperplane section $E$ of $S$. Notice that $E$ has degree $g+1$ and it can be assumed to be non-degenerate in a projective space of dimension $g-2$. Due to Castelnuovo's bound (see $\mathrm{A}-\mathrm{C}-\mathrm{G}-\mathrm{H}$, p.116) we have that $p_{a}(E) \leq 3$. Due to Lemma 5.1 such a rational map induces a morphism $\alpha: C \rightarrow E$ of degree 2 . Since $C$ is neither rational nor hyperelliptic, it follows that $p_{a}(C) \geq 2$.

If $p_{a}(E)=3$, then $E$ is smooth and the singular locus of $S$ must have dimension 0. Moreover, $\alpha: C \rightarrow E$ is a double cover; thus $\omega_{C}=\alpha^{*} \mathcal{L}$ for a suitable $\mathcal{L} \in \operatorname{Pic}(E)$ of degree $g-1$. Due to the definition of $\alpha$, there exists a canonical divisor $K$ without double points which is cut out on $C_{c a n}$ by $g-1$ pairwise distinct lines $L_{1}, \ldots, L_{g-1}$. Since the canonical system is cut out on $C_{c a n}$ by the hyperplanes, there exists a hyperplane $H \subseteq \mathbb{P}_{k}^{g-1}$ such that $H \cdot C_{\text {can }}=K$ in $\operatorname{Pic}(C)$. Notice that such an $H$ intersects each line $L_{i}$ at two points; thus it must contain it, whence $H \cap S=L_{1} \cup \cdots \cup L_{g-1} \cup D$, where $D$ is a curve on $S$ of degree 2. The irreducible components of $D$ are smooth and rational.

Let $D_{0}$ be one of them. Since $H$ cuts out on $C_{c a n}$ a canonical divisor, necessarily $C_{\text {can }} \cap D_{0}=\emptyset$. Since the singular locus of $S$ has dimension 0, the rational map $\widetilde{S} \rightarrow E$ defined above induces a well-defined morphism $D_{0} \rightarrow E$. Such a map cannot be constant, since $C_{c a n} \cap D_{0}=\emptyset$. This is absurd, since $E$ is not unirational.

We conclude that $p_{a}(E)=2$. It follows that there exists a map $\beta: E \rightarrow \mathbb{P}_{k}^{1}$ of degree 2 ; thus $\varrho:=\beta \circ \alpha$ would induce a $g_{4}^{1}$ on $\widetilde{C}$. Such a $g_{4}^{1}$ is cut out on $C_{\text {can }}$ by a pair of lines contained in $S$.

Let $\varrho: C \rightarrow \mathbb{P}_{k}^{1}$ be the cover of degree 4 associated to the aforementioned $g_{4}^{1}$. Recall that $\varrho$ factors through an embedding $i: C \rightarrow \mathbb{P}=\mathbb{P}(\mathcal{E})$ followed by the natural projection $\pi: \mathbb{P} \rightarrow \mathbb{P}_{k}^{1}$. Moreover, the canonical system on $C$ is cut out by the linear system $|\xi-2 f|$. Thus $C_{\text {can }}$ is contained in the threefold $\Sigma$ ruled in planes which is the image of $\mathbb{P}$ via $|\xi-2 f|$. The planes in $\Sigma$ cut out on $C_{\text {can }}$ the $g_{4}^{1}$.

If any one of the curves in $S$ cutting the $g_{4}^{1}$ were a pair of skew lines, then it could not cut out on $C_{c a n}$ a divisor lying in a plane. It follows that such curves must be (possibly reducible) conics; hence they are also contained in the fibres of $\Sigma$. We thus conclude that $S \subseteq \Sigma$.

Let $\tau$ be the tetragonality of $\varrho$. Since $S \subseteq \Sigma$ and $\operatorname{deg}(S)=g+1$, it follows from Theorem 2.4 again that either $g+1=g-1+\tau$, whence $\tau=2$, or $g+1 \geq 2 g-6-\tau$, whence $\tau \geq g-7$.

In the second case, since $\tau \leq(g-5) / 2$ (see Remark $2.3(1)$ ), we obtain $g \leq 9$, whence $\tau \leq 2$. The case $\tau<2$ cannot occur since, otherwise, $C_{\text {can }}$ would be contained in a surface of degree $g-1-\tau<g+1$. We conclude that $\tau=2$ in both cases. In particular, the invariant $\beta$ is $(6, g-3)$ with $g-3 \geq 6$, i.e., $g \geq 9$. If $g \geq 16$, then $\tau<(g-9) / 3$; hence the $g_{4}^{1}$ is composed with an involution onto a curve of genus 2 (see Proposition 2.5). Finally, since $\tau=2>0$, Remark 2.3 (5) guarantees the uniqueness of the $g_{4}^{1}$ on $C$. 
We can summarise the results of this section in the following.

Proposition 5.4. Let $C$ be a non-hyperelliptic curve of genus $g$ and let $g+1$ be the minimal degree of a surface $S \subseteq \check{\mathbb{P}}_{k}^{g-1}$ containing $C_{\text {can }}$.

If $C$ does not carry any $g_{7}^{2}$, then $g \geq 9$ and $C$ is endowed with a unique $g_{4}^{1}$ with tetragonality $\tau=2$. If $g \geq 16$ such a $g_{4}^{1}$ is composed with an involution on a curve of genus 2. The surface $S$ is a conic bundle onto $\mathbb{P}_{k}^{1}$ whose fibres cut out on the canonical model of $C$ a $g_{4}^{1}$.

\section{Theorem D: Its proof And some Consequences}

We start this section by proving the Main Theorem stated in the introduction.

Proof of Theorem D. Let $C$ be a curve of genus $g$ whose canonical model lies on a surface of degree $g+1$ but not on surfaces of lesser degree. In Propositions 3.3, 4.4 and 5.4 above we showed that either $C$ is tetragonal with $g \geq 9$ (and tetragonality $\tau=2$ ) or it is birationally isomorphic to a plane septic curve with at most double points as singularities and $7 \leq g \leq 15$. It remains to prove that a curve cannot fall simultaneously in the two aforementioned classes.

To this purpose it suffices to prove that the gonality of a plane septic curve of genus $g \geq 9$ and with at most double points as singularities cannot be 4 . This is well-known when the double points are nodes or ordinary cusps (see [C-K1] and C-K2]). Since infinitely near points can occur in our setup we need to make use of some more refined results proved in $\mathrm{O}-\mathrm{S}$. Indeed, Proposition 2 of $\mathrm{O}-\mathrm{S}$ ] can be easily applied with $d=7, \nu=2, \delta=15-g \leq 6$ in order to show that the gonality of the plane septic curve we are interested in is exactly 5 and not 4 .

We dedicate the rest of this section to a corollary and some remarks.

Corollary 6.1. Let $C$ be a non-hyperelliptic curve of genus $g$. If $C_{c a n}$ is contained in a surface in $\mathbb{P}_{k}^{g-1}$ of degree $g+1$, then $C$ is either trigonal or tetragonal or pentagonal or isomorphic to a plane septic curve. If $C$ is pentagonal, then $7 \leq g \leq$ 14 .

Proof. Let $S \subseteq \check{\mathbb{P}}_{k}^{g-1}$ be a surface of minimal degree containing $C_{\text {can }}$.

We know that $\operatorname{deg}(S) \leq g+1$. If $d=g-2$, then $C$ is trigonal (Theorem A). If $\operatorname{deg}(S)=g-1, g$, then $C$ is either tetragonal or isomorphic to a plane sextic, whence it is pentagonal and $g=10$ (Theorems B and C). If $\operatorname{deg}(S)=g+1$, then $C$ is either tetragonal (Theorem D ii)) or it is isomorphic to a plane septic (Theorem D i) with $g=15)$ or it is birationally isomorphic to a plane septic with at least a double point; then it is pentagonal (Theorem $\mathrm{D}$ i) with $7 \leq g \leq 14$ ).

If $C$ is isomorphic to a plane septic, then it cannot carry any $g_{d}^{1}$ with $d \leq 5$ (see A-C-G-H], Appendix A, Ex. 18).

Let $C$ be a curve of genus $g$ whose canonical model lies on a surface $S$ of degree $g+1$. Let us now examine the possible values for the scrollar invariant $\alpha$.

Remark 6.2. When the $g_{4}^{1}$ is composed with an involution onto a curve of genus 2 (e.g. if $g \geq 16$ ); then $\alpha=\left(3, \alpha_{2}, g-\alpha_{2}\right)$ (due to Remark 2.3 (4)). Moreover, $3+\alpha_{2} \geq g-\alpha_{2}$ (see [Ch, Proposition 3.3); thus we finally obtain that $\alpha_{2} \geq(g-3) / 2$. 
In particular, we have the following four possible cases for the scrollar invariant $\alpha$ : $(6.2 .1)$

$$
\left(3, \frac{g-3}{2}, \frac{g+3}{2}\right), \quad\left(3, \frac{g-2}{2}, \frac{g+2}{2}\right), \quad\left(3, \frac{g-1}{2}, \frac{g+1}{2}\right), \quad\left(3, \frac{g}{2}, \frac{g}{2}\right) .
$$

Obviously only two of them are possible for a fixed value of $g$, according with its parity. Moreover, such cases actually occur (see $[\mathrm{B}-\mathrm{C}-\mathrm{F}]$ for the details).

Remark 6.3. The picture when $9 \leq g \leq 15$ is richer since many other cases are possible for the scrollar invariant $\alpha$. Recall that in any case we have $\beta=(6, g-3)$. Taking into account Remark 2.3 (1) and that $\alpha_{1}+\alpha_{2} \geq \alpha_{3}$ (see [Ch, Proposition 3.3), then $\alpha$ is one of the following:

$$
\begin{aligned}
& g=9) \quad(4,4,4),(3,4,5),(3,3,6) ; \\
& g=10)(4,4,5),(3,5,5),(3,4,6) ; \\
& g=11)(4,5,5),(4,4,6),(3,5,6),(3,4,7) ; \\
& g=12)(5,5,5),(4,5,6),(4,4,7),(3,6,6),(3,5,7) ; \\
& g=13)(5,5,6),(4,6,6),(4,5,7),(4,4,8),(3,6,7),(3,5,8) ; \\
& g=14)(5,6,6),(5,5,7),(4,6,7),(4,5,8),(3,7,7),(3,6,8) ; \\
& g=15)(6,6,6),(5,6,7),(5,5,8),(4,7,7),(4,6,8),(4,5,9),(3,7,8),(3,6,9) .
\end{aligned}
$$

On the one hand it is not difficult to show (see e.g. $[\mathrm{Br}$, Proposition 3.1) that the cases

$$
(4,4,7), \quad(4,4,8), \quad(5,5,7), \quad(4,5,8), \quad(5,5,8), \quad(4,7,7), \quad(4,5,9)
$$

never occur, since $C$ is smooth. On the other hand, Theorem 2.2 guarantees that the cases

$$
(4,4,4), \quad(4,4,5), \quad(4,5,5), \quad(4,4,6), \quad(5,5,5), \quad(5,5,6), \quad(6,6,6)
$$

actually occur. Finally, taking double covers of hyperelliptic curves of genus 2 branched along suitable divisors of degree $2 g-6$ it is possible to show that also all the cases with $\alpha_{1}=3$ occur (see $[\mathrm{B}-\mathrm{C}-\mathrm{F}$. for the details).

It could be, perhaps, interesting to deal with the remaining cases, namely

$$
(4,5,6), \quad(4,6,6), \quad(4,5,7), \quad(5,6,6), \quad(4,6,7), \quad(5,6,7), \quad(4,6,8) .
$$

Remark 6.4. Since the Brill-Noether number $\varrho(7,2,7)$ is 1 , it follows that each curve $C$ of genus $g=7$ is endowed with a morphism $f: C \rightarrow D \subseteq \mathbb{P}_{k}^{2}$ onto a nondegenerate integral curve $D$, where $7=\operatorname{deg}(f) \operatorname{deg}(D)$. It follows that $f$ is actually a birational morphism; thus $D$ is necessarily a singular septic curve. By adjunction the canonical system on $C$ is cut out on $D$ by the quartic adjoint curves in $\mathbb{P}_{k}^{2}$.

An argument similar to the one used in Section 4 then shows that the canonical model $C_{c a n}$ of each such curve is always contained in a surface of degree at most 8 . Moreover, we are now able to define a stratification

$$
\bigcup_{\ell=5}^{8} \mathfrak{D}_{7}(\ell)
$$

of the non-hyperelliptic part of the moduli space $\mathfrak{M}_{7}: \mathfrak{D}_{7}(\ell)$ is the locus of points corresponding to curves $C$ such that the minimal degree of a surface containing their canonical models $C_{\text {can }}$ is $\ell$.

More precisely we have that the points in $\mathfrak{D}_{7}(5)$ correspond to trigonal curves (see Theorem A: this is usually denoted by $\mathfrak{M}_{7,3}^{1}$ in the literature). The points in $\mathfrak{D}_{7}(6)$ correspond to curves which are either bielliptic or birationally isomorphic to a plane 
sextic curve with three (possibly infinitely near) double points (see Theorem B). The points in $\mathfrak{D}_{7}(7)$ correspond to tetragonal curves with scrollar invariants $\alpha=(3,3,4)$ and $\beta=(5,5)$. Finally we have the largest stratum $\mathfrak{D}_{7}(8)$ whose points correspond to curves which are isomorphic to a plane septic with eight (possibly infinitely near) double points.

\section{REFERENCES}

[A-C-G-H] E. Arbarello, M. Cornalba, P.A. Griffiths, J. Harris, Geometry of algebraic curves, vol. I, Springer, 1985. MR770932 (86h:14019)

[B-C-F] E. Ballico, G. Casnati, C. Fontanari, On the geometry of bihyperelliptic curves, J. Korean Math. Soc. 44 (2007), 1339-1350. MR2358958 (2008i:14040)

[B-C-N] E. Ballico, G. Casnati, R. Notari, Canonical curves with low apolarity, J. Algebra 332 (2011), 229-243. MR2774686

[Br] J. Brawner, Tetragonal curves, scrolls and K3-surfaces, Trans. Amer. Math. Soc. 349 (1997), 3075-3091. MR1401515 (97j:14056)

[Cs] G. Casnati, Canonical curves on surfaces of very low degree, Proc. Amer. Math. Soc. 140 (2012), 1185-1197.

[C-E] G. Casnati, T. Ekedahl, Covers of algebraic varieties I. A general structure theorem, covers of degree 3, 4 and Enriques surfaces, J. Algebraic Geom. 5 (1996), 439-460. MR:1382731 (97c:14014)

[Ch] G. Chaves, Revêtement ramifiés de la droite projective complexe, Math. Z. 226 (1997), 67-84. MR 1472141 (98j:14038)

[C-H] C. Ciliberto, J. Harris, Surfaces of low degree containing a general canonical curve, Comm. Algebra 27 (1999), 1127-1140. MR1669124 (2000c:14051)

[C-K1] M. Coppens, T. Kato, The gonality of smooth curves with plane models, Manuscripta Math. 70 (1990), 5-25. MR1080899(92a:14027a)

[C-K2] M. Coppens, T. Kato, Correction to the gonality of smooth curves with plane models, Manuscripta Math. 71 (1991), 337-338. MR1103738(92a:14027b)

[Co] I. Coşkun, Surfaces of low degree containing a canonical curve. To appear in Contemp. Math.

[De] M. Demazure, Surfaces de Del Pezzo - II, III, IV, V, Séminaire sur les singularités des surfaces, Palaiseau, France 1976-1977 (M. Demazure, H. Pinkham, B. Teissier, eds.), Lecture Notes in Math. 777, Springer, 1980.

[Ha] R. Hartshorne, Algebraic geometry, Springer, 1977. MR0463157 (57:3116)

[Mi] J.C. Migliore, Introduction to liaison theory and deficiency modules, Progress in Mathematics, vol. 165, Birkhäuser, 1998. MR1712469 (2000g:14058)

[O-S] M. Ohkouchi, F. Sakai, The gonality of singular plane curves, Tokyo J. Math. 27 (2004), 137-147. MR2060080(2005d:14046)

[SD] B. Saint-Donat, On Petri's analysis of the linear system of quadrics through a canonical curve, Math. Ann. 206 (1973), 157-175. MR0337983 (49:2752)

[Sch1] F.O. Schreyer, Syzygies of canonical curves and special linear series, Math. Ann. 275 (1986), 105-137. MR849058 (87j:14052)

[Sch2] F.O. Schreyer, A standard basis approach to syzygies of canonical curves, J. Reine Angew. Math. 421 (1991), 83-123. MR.1129577 (92j:14040)

[Za] O. Zariski, A simplified proof for the resolution of singularities of an algebraic surface, Ann. of Math. (2) 43 (1942), 583-593. MR0006851 (4:52c)

Dipartimento di Matematica, Politecnico di Torino, C. so Duca degli Abruzzi 24, 10129 Torino, Italy

E-mail address: casnati@calvino.polito.it 